\title{
Fractional quantum Hall states in the vicinity of Mott plateaus
}

\author{
R. O. Umucalılar ${ }^{1,2, *}$ and Erich J. Mueller ${ }^{2}$ \\ ${ }^{1}$ Department of Physics, Bilkent University, 06800 Ankara, Turkey \\ ${ }^{2}$ Laboratory for Atomic and Solid State Physics, Cornell University, Ithaca, New York 14853, USA
}

(Received 22 February 2010; published 24 May 2010)

\begin{abstract}
We perform variational Monte Carlo calculations to show that bosons in a rotating optical lattice will form analogs of fractional quantum Hall states when the tunneling is sufficiently weak compared to the interactions, and the deviation of density from an integer is commensurate with the effective magnetic field. We compare the energies of superfluid and correlated states to one another and to the energies found in full configuration-interaction calculations on small systems. We look at overlaps between our variational states and the exact ground state, characterizing the ways in which fractional quantum Hall effect correlations manifest themselves near the Mott insulating state. We explore the experimental signatures of these states.
\end{abstract}

DOI: 10.1103/PhysRevA.81.053628

PACS number(s): 03.75.Lm, 03.75.Hh, 73.43.-f

\section{INTRODUCTION}

We consider the interplay between three paradigmatic quantum states of bosons in rotating lattices: Mott insulators, superfluids, and fractional quantum Hall states. The Mott insulator is found when there is an integer number of particles per lattice site, and the tunneling is sufficiently suppressed relative to the interactions. It is an incompressible state, where interactions freeze the particles in place. In the standard cartoon, when the density of such a system is tuned away from commensurability, the excess particles (or holes) "skate" across the frozen Mott sea, forming a superfluid. If the system is rotating, one expects that the collective motion of this superfluid will produce a vortex lattice. In 2007, Umucalilar and Oktel [1] argued that, when the rotation rate is high enough that the number of vortices is comparable to the number of excess particles, then the superfluid will be unstable to forming a correlated state of matter with particles bound to vortices-a situation analogous to that found in the fractional quantum Hall state. They supported this argument by estimating the energy of the superfluid and the correlated state. Here we confirm this scenario through more rigorous calculations. By using Monte Carlo techniques, we compare the energy of variational states describing fractional quantum Hall states and superfluid vortex lattices with each other. We also compare these energies with exact results calculated for small numbers of particles. We find that there is a range of parameters for which the fractional quantum Hall states are more favorable than superfluid states. We note, however, that the energy differences between these states scales as the tunneling energy and can be quite small.

Most previous studies of analogs of fractional quantum Hall states in optical lattices have focused on the low-density limit, where there are much fewer than one particle per site. In the context of cold atoms, Hafezi et al. [2] gave an excellent review of the basic physics of this limit (including symmetry and topology arguments), and argued that one can continuously deform a Mott insulating state into a fractional quantum Hall state by varying the strength of an additional superlattice potential [3]. They also proposed using Bragg spectroscopy to probe these states. Palmer et al. [4] performed a number

*onur@fen.bilkent.edu.tr of calculations focused on the role of the trap, detection schemes, and inhomogeneities that can spontaneously appear in these systems. Bhat et al. [5] carried out full configurationinteraction calculations for a small number of particles in a rotating lattice with hard-wall boundary conditions. Möller and Cooper analyzed the relevance of composite fermion wave functions to describing these systems [6]. Cooper recently produced a review of the physics of rotating cold atom clouds including analogs of the quantum Hall effect in lattices [7]. These, and our present study, build on initial works motivated by solid state systems [8].

Translation of these arguments to higher densities is not completely trivial. The superfluid near the Mott state is more complicated than the standard cartoon suggests. For example, the mean-field description treats it as a two-component plasma of particles and holes, with a small imbalance between the densities of particles and holes. Despite these complications, we find that, when the deviation of the particle density from an integer value is commensurate with the magnetic flux, one can indeed see analogs of the fractional quantum Hall effect.

We start our analysis with the well-known Bose-Hubbard Hamiltonian in an effective magnetic field,

$$
H_{0}=-t \sum_{\langle i, j\rangle} a_{i}^{\dagger} a_{j} e^{i A_{i j}}+\frac{U}{2} \sum_{i} \hat{n}_{i}\left(\hat{n}_{i}-1\right),
$$

where $a_{i}\left(a_{i}^{\dagger}\right)$ is the bosonic annihilation (creation) operator at site $i$ and $\hat{n}_{i}=a_{i}^{\dagger} a_{i}$ is the number operator. The tunneling is parametrized by $t$ and on-site interactions by $U$. We use the Landau gauge $\mathbf{A}=(-B y, 0)$, so the phases $A_{i j}=$ $\exp \left(i e / \hbar c \int_{\mathbf{r}_{j}}^{\mathbf{r}_{i}} \mathbf{A} \cdot d \mathbf{l}\right)$ acquired when hopping in the $\pm x$ direction are $\mp 2 \pi \alpha i_{y}$, where $i_{y}$ is the $y$ coordinate scaled by lattice constant $a$, and in the $y$ direction $A_{i j}=0$. Here, $\alpha=B a^{2} /(h c / e)=p / q$ is the flux quantum per plaquette, and we take $p$ and $q$ to be relatively prime integers. The singleparticle spectrum for this problem is the famous Hofstadter butterfly [9]. The phase boundary between the Mott insulator and superfluid carries signatures of this single-particle physics [1,10-12]. Away from the tips of the Mott lobes, the physics of the superfluid-Mott transition of the nonrotating system is in the universality class of the dilute Bose gas. Thus we expect that phenomena which can be seen in the dilute Bose gas will 
occur there, including the analogs of fractional quantum Hall physics which we are exploring here.

\section{VARIATIONAL WAVE FUNCTION}

\section{A. Laughlin state}

We consider the variational ansatz

$$
|\Psi\rangle=\sum_{z_{1}, \ldots, z_{N}} \psi\left(z_{1}, \ldots, z_{N}\right) a_{z_{1}}^{\dagger} \cdots a_{z_{N}}^{\dagger}\left|\Psi_{\mathrm{MI}}\right\rangle,
$$

where $\left|\Psi_{\mathrm{MI}}\right\rangle=\prod_{j}\left(a_{j}^{\dagger}\right)^{n_{0}} / \sqrt{n_{0}}$ ! $|\mathrm{vac}\rangle$ is the Mott insulator state with $n_{0}$ particles per site and $\psi$ is the Laughlin wave function [13] with filling $v=1 / m$. To describe bosons, $m$ must be even. The complex coordinate $z_{i}=x_{i}+i y_{i}$ specifies the location of the $i$ th particle, with $i$ running from 1 to $N$, where $N$ is the number of excess particles. The sum over $z_{i}$ is a sum over all lattice sites. To describe a state with excess holes, we replace $a^{\dagger}$ with $a$.

To minimize the role of boundaries, the model in (1) is typically solved either on a sphere or a torus [8,14]. We will work in an $L \times L$ torus geometry, corresponding to quasiperiodic boundary conditions

$$
\begin{gathered}
\psi\left(\ldots, z_{k}+L, \ldots\right)=\psi\left(\ldots, z_{k}, \ldots\right), \\
\psi\left(\ldots, z_{k}+i L, \ldots\right)=e^{-i(2 \pi m N / L) x_{k}} \psi\left(\ldots, z_{k}, \ldots\right) .
\end{gathered}
$$

For these boundary conditions, the Laughlin wave function can explicitly be written as [14]

$$
\begin{aligned}
& \psi\left(z_{1}, \ldots, z_{N}\right) \\
& =\mathcal{N} \exp \left(i K_{x} \sum_{i} x_{i}\right) \exp \left(-K_{y} \sum_{i} y_{i}\right) \\
& \quad \times \exp \left(-\frac{\pi m N}{L^{2}} \sum_{i} y_{i}^{2}\right) \prod_{\beta=1}^{m} \vartheta_{1}\left(\left(Z-Z_{\beta}\right) \frac{\pi}{L}\right) \\
& \quad \times \prod_{i<j}^{N}\left[\vartheta_{1}\left(\left(z_{i}-z_{j}\right) \frac{\pi}{L}\right)\right]^{m} .
\end{aligned}
$$

Here, $\mathcal{N}$ is the normalization factor, $Z=\sum_{i} z_{i}$ is $N$ times the center-of-mass coordinate, and $Z_{\beta}=X_{\beta}+i Y_{\beta}$ are the $a$ priori arbitrary locations of the center-of-mass zeros. To satisfy the boundary conditions, one requires $\sum_{\beta} X_{\beta}=n_{1} L\left(n_{1} \in\right.$ $\mathbb{Z}), K_{x}=2 \pi n_{2} / L\left(n_{2} \in \mathbb{Z}\right)$, and $K_{y}=-2 \pi \sum_{\beta} Y_{\beta} / L^{2}$. The quasiperiodic Jacobi $\vartheta$ functions are defined by

$$
\vartheta_{1}\left(z, e^{i \pi \tau}\right)=\sum_{-\infty}^{\infty}(-1)^{n-1 / 2} e^{i \pi \tau(n+1 / 2)^{2}} e^{(2 n+1) i z} .
$$

For our square geometry $\tau=i$. This function is odd with respect to $z$ and has the following quasiperiodicity properties: $\vartheta_{1}(z+\pi)=-\vartheta_{1}(z)$ and $\vartheta_{1}(z+\tau \pi)=-e^{-i \pi \tau} e^{-2 i z} \vartheta_{1}(z)$. The relation between the flux quantum per plaquette, $\alpha=$ $N_{\phi} / L^{2}$, filling fraction $v=N / N_{\phi}$, and excess particle density $\varepsilon=N / L^{2}$ is succinctly given by $\alpha \nu=\varepsilon$, where $N_{\phi}$ denotes the number of flux quanta in the $L \times L$ lattice we consider. In what follows, we will restrict ourselves to the $v=1 / 2$ Laughlin state $(m=2)$, so that the commensurability requirement between the magnetic flux and particle density becomes $\alpha=2 \varepsilon$.

\section{B. Superfluid state}

We will compare the Laughlin state introduced in Sec. II A with a Gutzwiller mean-field state

$$
\left|\Psi_{\mathrm{MF}}\right\rangle=\prod_{i}\left(\sum_{n} f_{n}^{i}|n\rangle_{i}\right)
$$

where $f_{n}^{i}$ are variational parameters. This wave function is commonly used to describe the superfluid in the Bose-Hubbard model [15]. It is exact in the noninteracting limit and captures the effect of number squeezing. Its main deficit is that it does not capture any of the short-range correlations in the superfluid. Regardless, the energies it produces are good estimates of the superfluid energy. In the nonrotating case, the superfluid is translationally invariant, and the coefficients $f_{n}^{i}$ are independent of $i$. In our case, where the lattice is rotating, a vortex lattice forms, breaking translational invariance.

Near the Mott lobe, the site occupations are dominated by $n=n_{0}$ and $n=n_{0} \pm 1$ : that is, it is extremely unlikely to have more than one extra particle or hole on a given site. We therefore truncate our basis to only these three values of $n$. This will also facilitate direct comparison with configurationinteraction calculations using the same truncated basis. We work in an $L \times L$ lattice, using the boundary conditions which are equivalent to those in Eq. (3).

The numerical techniques for optimizing the $f_{n}^{i}$ are well documented [10], and we will not repeat the detailed discussion here. These can be described in terms of a variational calculation where one minimizes $\left\langle\Psi_{\mathrm{MF}}\left|H_{0}\right| \Psi_{\mathrm{MF}}\right\rangle$ with the constraints that the total number of particles $M$ and normalization $\left\langle\Psi_{\mathrm{MF}} \mid \Psi_{\mathrm{MF}}\right\rangle$ are fixed: this involves introducing the chemical potential $\mu$ and a number of other Lagrange multipliers. In practice, it is more convenient to write $H=H_{0}-\mu M$ and follow an iterative procedure based upon mean-field theory. These two approaches are completely equivalent. In comparing energies with our other variational state, one must be cautious and be sure to use $\left\langle H_{0}\right\rangle=\langle H\rangle+\mu M$.

\section{EXACT RESULTS ON SMALL SYSTEMS}

\section{A. Approach and results}

For small systems, we can exactly diagonalize the Hamiltonian in Eq. (1), taking a configuration-interaction approach where we truncate the allowed number of particles on a given site to be $n_{0}, n_{0}-1$, or $n_{0}+1$. For definiteness we take $n_{0}=1$; changing this value just scales the hopping matrix elements $t$. For these small system sizes, we can also directly calculate $\left\langle\Psi\left|H_{0}\right| \Psi\right\rangle$. In Sec. IV we will discuss larger systems where we need to resort to a Monte Carlo algorithm for calculating this energy.

We consider 12 particles in a $3 \times 3$ lattice, so that the excess particle density is $1 / 3$. We take $v=1 / 2$ and accordingly the number of quanta of flux per plaquette is $\alpha=2 / 3$. Figure 1 displays the energies (measured in units of $U$ ) of the first few hundred exact energy eigenstates together with the energies of our two variational wave functions Eqs. (2) and (5). We emphasize that our ansatz for the fractional quantum Hall state is not just the Laughlin state, where flux is bound to each particle, but is rather the coexistence of a Mott state and a Laughlin state, with flux bound only to the excess particles. 


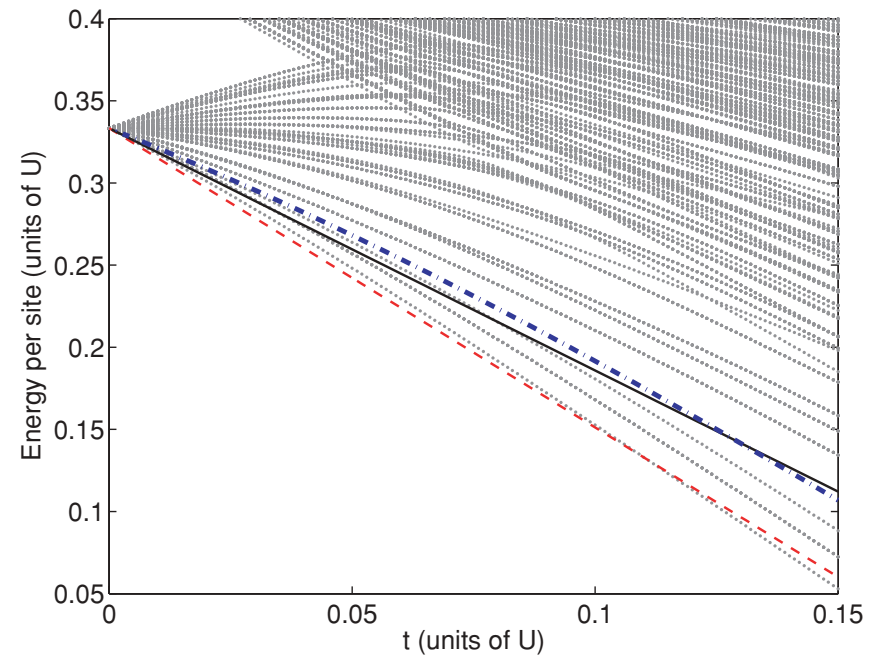

FIG. 1. (Color online) Exact many-body spectrum for 12 particles in a $3 \times 3$ lattice with $\alpha=2 / 3$, considering only 0,1 , and 2 atoms per site (for $v=1 / 2$, the excess particle density is $\varepsilon=\alpha v=1 / 3$ ). Also shown by the solid black line is our variational estimate of the energy of a fractional quantum Hall state of excess particles in the presence of a Mott background. The dash-dotted blue line shows the Gutzwiller mean-field superfluid energy for the same density $(1+\varepsilon)$, corresponding to a vortex lattice where the cores are filled with Mott insulator. The dashed red line is the estimate of the ground-state energy from Eq. (6), first introduced in [1]. For low enough $t$, the variational energy of the correlated state of excess particles is lower than the superfluid energy.

In Fig. 1 we also show the estimate from Ref. [1], which is supposed to describe the correlated state near the Mott insulator,

$$
\Delta E=U n_{0} \varepsilon-t\left(n_{0}+1\right) f(\alpha) \varepsilon,
$$

where the first term represents the on-site interaction of excess particles with the Mott insulator and the second term is the hopping energy of particles in the Hofstadter ground state denoted by $-t f(\alpha), f(\alpha)>0$ being the dimensionless maximum eigenvalue of the Hofstadter spectrum. Note that $t$ is enhanced by a factor of $\left(n_{0}+1\right)$ owing to the Mott background. No interaction energy is included, as it is expected that in this regime the excess atoms avoid one another. It is remarkable how closely this estimate matches the results of the exact diagonalization for small $t$.

For $t \lesssim 0.13$ the energy of our candidate fractional quantum Hall state (with optimized $Z_{\beta}$ ) is lower than that of the superfluid, while the opposite holds for larger $t$. Our physical picture of this is that as $t$ grows the Mott insulator melts, and the density of mobile atoms is no longer commensurate with the magnetic field.

For very small $t$, the fractional quantum Hall state's energy agrees very well with the exact ground-state energy; this is shown more clearly in Fig. 2(b). In Fig. 2(a) we show the overlap between our variational state and the exact ground state. At low $t$ the overlap is greater than $95 \%$, but it falls off with increasing $t$, presumably because of the increasing importance of particle-hole excitations. The overlap between the ground state and the mean-field superfluid [inset of Fig. 2(a)] is never large, and their energies in Fig. 1 never
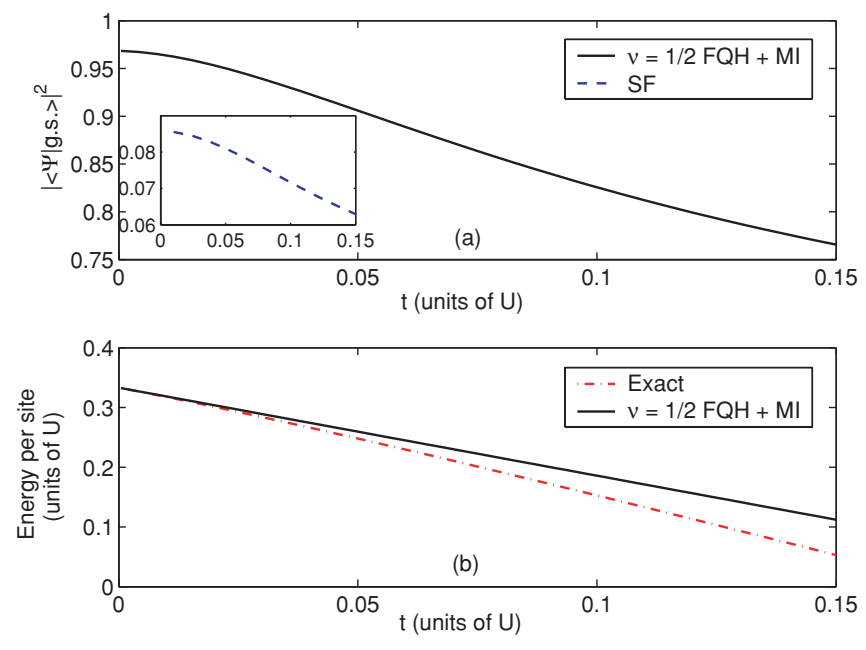

FIG. 2. (Color online) (a) Overlap between the $v=1 / 2 \mathrm{FQH}+$ MI state $[|\Psi\rangle$ from Eq. (2)] and the exact ground state [|g.s. $\rangle$, determined from diagonalizing Eq. (1) in a truncated basis] as a function of tunneling strength $t$, using the same parameters as in Fig. 1. Also shown in the inset is the overlap between a superfluid vortex lattice and $\mid$ g.s. $\rangle$. (b) Comparison of the variational and exact energies from Fig. 1.

approach one another. We believe this is due in part to the fact that the mean-field state breaks translational invariance and consequently involves a superposition of many eigenstates [16]. A quantum superposition of vortex lattices may in fact be a good alternative description of the fractional quantum Hall (FQH) state.

Given the small difference between the energies of our two variational states, one must be somewhat cautious about ascribing too much significance to the crossing at $t \sim 0.13$. One also might be concerned that at that value of $t$ both variational states have an energy that is significantly higher than that of the ground state, suggesting that neither may be particularly good descriptions of the true ground state. A third concern is that there is no sign of a phase transition in Fig. 2(a); the overlap between the fractional quantum Hall state and the exact ground state remains above $75 \%$ out to $t \sim 0.15$. Despite these caveats, the large overlap at small $t$ is convincing evidence that the ground state at low $t$ is a fractional quantum Hall state of excess particles, and it would be surprising if the system formed a correlated state at large $t$.

\section{B. Variational parameters}

In Fig. 3 we show how the energy of the variational state depends on the parameters $Z_{\beta}$, which represent where "vortices" can be found around which the center of mass flows. The boundary conditions in Eq. (3) force the wave function to have $m=1 / v$ of these zeros (in the present case $m=2$ ). In the absence of the lattice, the energy is invariant under change in these parameters, leading to an $m$-fold degeneracy of the ground state [17]. Here, this symmetry is absent and the energy depends on $Z_{\beta}$. Not surprisingly, the overlap between the variational state and the exact ground state is directly correlated with the energy. This overlap has a maximum when the variational energy has a minimum. 

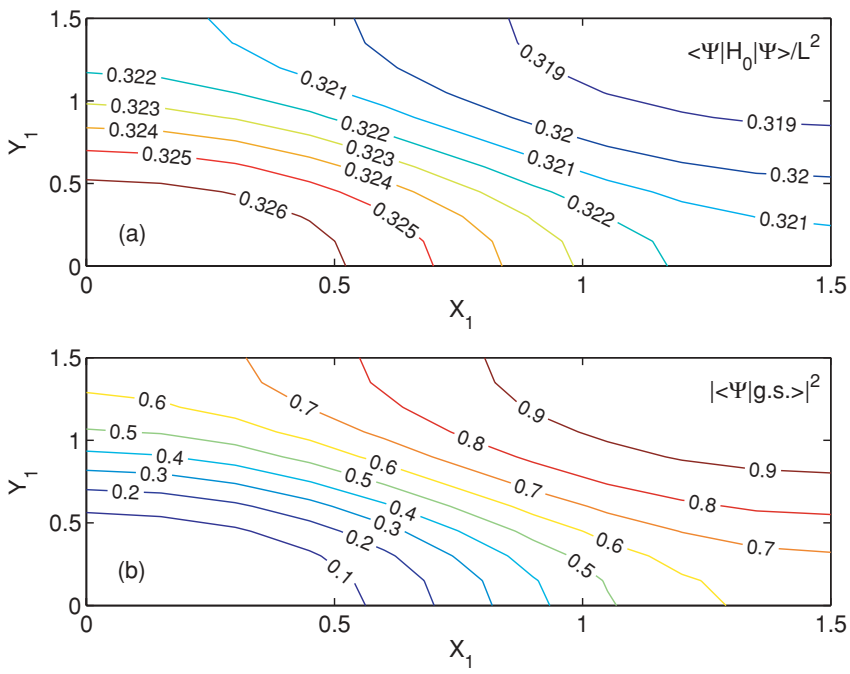

FIG. 3. (Color online) Variational energy (in units of $U$ ) (a) and the overlap with the exact ground state (b) as a function of center-of-mass zeros $Z_{1}=X_{1}+i Y_{1}, Z_{2}=L-Z_{1}$, measured in units of the lattice constant. As with Fig. 1, we consider an $L \times L$ cell with $L=3$, flux per plaquette, $\alpha=2 / 3$, filling factor $v=1 / 2$, and total particle number $M=12$. We take $K_{x}=0$, $K_{y}=0$, and $t=0.01 U$. The lower the variational energy, the higher the overlap. The lowest energy occurs for $X_{1}=Y_{1}=L / 2$, where the overlap is $96.4 \%$. At this point, the variational energy is $0.3186 U$, which is very close to the exact ground-state energy of $0.3176 U$.

\section{VARIATIONAL MONTE CARLO METHOD}

Unfortunately, the maximum size of the system that can be treated by the techniques of Sec. III is quite limited. Our preceding results for small system size predominantly serve as a guide for physical intuition and cannot quantitatively describe the physics of the infinite system. Here we introduce a variational Monte Carlo (VMC) algorithm [18] in order to calculate the energy $\langle\Psi|H| \Psi\rangle=\left\langle\Psi\left|H_{0}-\mu M\right| \Psi\right\rangle$, where $M$ is the total number of particles. This will allow us to make a more solid comparison of the energies of the superfluid and correlated states and draw the phase diagram in Fig. 4. This phase diagram illustrates the regions of the $t-\mu$ plane where either the superfluid or correlated state has a lower energy.

We begin by introducing a basis $\left|R=\left\{z_{1}, \ldots, z_{N}\right\}\right\rangle$ where the $N$ excess particles are at sites $z_{1}$ through $z_{N}$. This allows us to write

$$
\begin{gathered}
\langle\Psi|H| \Psi\rangle=\sum_{R R^{\prime}}\langle\Psi \mid R\rangle\left\langle R|H| R^{\prime}\right\rangle\left\langle R^{\prime} \mid \Psi\right\rangle=\sum_{R} P_{R} E_{R}, \\
P_{R}=|\langle R \mid \Psi\rangle|^{2}, \quad E_{R}=\sum_{R^{\prime}} \frac{\left\langle R|H| R^{\prime}\right\rangle\left\langle R^{\prime} \mid \Psi\right\rangle}{\langle R \mid \Psi\rangle} .
\end{gathered}
$$

We use a Metropolis algorithm to sample the sum over $R$. Starting from some configuration $R_{0}$, we generate a new one $R_{1}$ by attempting to move a single particle by one site. We accept the move with probability $\min \left\{1, P_{R_{1}} / P_{R_{0}}\right\}$; we then continue the procedure to generate $R_{2}, R_{3}, \ldots$ In the resulting Markov chain, each configuration $R$ will appear with probability $P_{R}$. After $S$ steps, the energy is then estimated

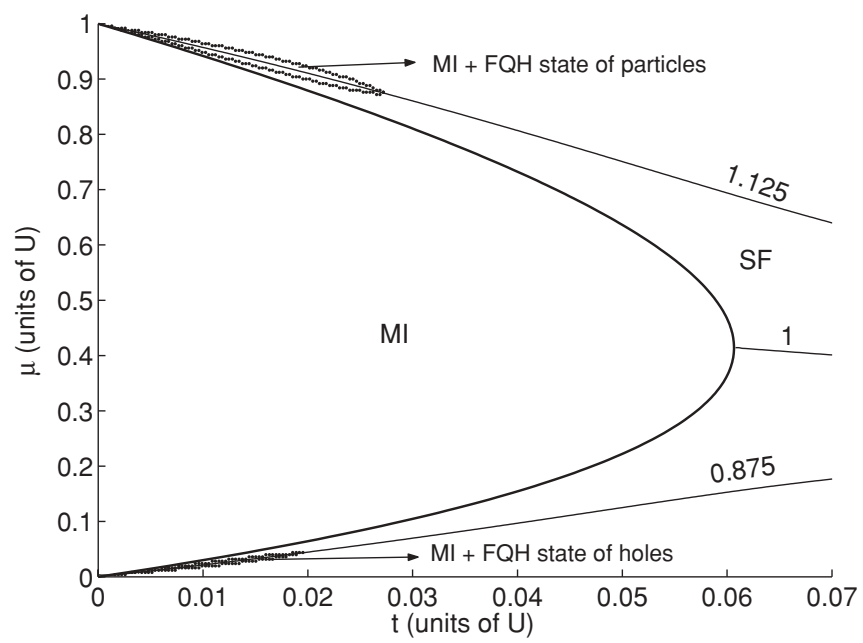

FIG. 4. Phase diagram for $\alpha=1 / 4$ and $v=1 / 2$. Boundary between Mott insulator (MI) and superfluid (SF) states is found from a mean-field calculation. Excess particle (or hole) density is $\varepsilon=\alpha \nu=0.125$. The boundary of the coexistent $v=1 / 2 \mathrm{FQH}$ state of excess particles (holes) and $n_{0}=1 \mathrm{MI}$ state centered around the $1.125(0.875)$ constant-density line is determined from a comparison of VMC and mean-field energies. We consider eight particles in an $8 \times 8$ lattice in the VMC calculation.

as $E_{S}=\sum_{i=1}^{S} E_{R_{i}} / S$. As is usual, we discard the first few thousand steps so as not to bias the sum by our choice of initial configuration. We use a binning analysis to estimate the statistical error on our sum [19].

For each $R$, we calculate $E_{R}$ directly. The Hamiltonian connects only a finite number of different configurations (those that differ by moving one particle by one site), and the sum is straightforward numerically.

As a further simplification we note that $E(\mu, t)=$ $E_{0}(\mu)-\left(1+n_{0}\right) t K$, where $E_{0}=U n_{0} \varepsilon+U\left(n_{0}-1\right) n_{0} / 2-$ $\mu\left(n_{0}+\varepsilon\right)$ is the expectation value of the on-site terms in $H$ and $-\left(1+n_{0}\right) t K$ is the expectation value of the hopping energy. $K$ is independent of $n_{0}$, as the only role of the Mott background is to provide a Bose-enhancement term of $\left(1+n_{0}\right)$. By using the Monte Carlo algorithm to calculate $K$, rather than $E$, we produce $E(\mu, t)$ for all $n_{0}, \mu$, and $t$ at once.

Table I lists the parameters for which we have performed VMC calculations. For the smallest system sizes $(L=3,4,5)$, we find that the VMC results agree with the direct calculation of the variational energy. From the table, we conclude that finite-size effects are significant in the $L=3$ cases, but for larger $L$ the differences between the energies of the two systems are within a few percent. We have not extrapolated to $L=\infty$.

Figure 4 illustrates our results for $\alpha=1 / 4$. Near the constant-density line $n=1+\varepsilon$, there is a region where our variational wave function has a lower energy than the Gutzwiller mean-field vortex lattice. This corresponds to an incompressible $v=1 / 2$ bosonic Laughlin state above the $n_{0}=1$ Mott insulator. The same argument can be advanced for holes by just exchanging the creation operators in Eq. (2) with annihilation operators, leading to a coexisting Mott insulator and FQH state of holes near the $1-\varepsilon$ line, although it is less visible than in the particle case. 
TABLE I. Results of our variational Monte Carlo calculation. $\alpha$ is the flux number per plaqette, $\varepsilon$ is the density of excess particles, $L$ is the system size, $N$ is the number of excess particles, and $-\left(1+n_{0}\right) t K$ is the hopping energy per site, where $t$ is the hopping matrix element and $n_{0}$ is the number of particles per site in the underlying Mott state. $K$ is dimensionless. Our estimates of the statistical error in $K$ from a binning analysis of 80000 samples are given by $\delta K$.

\begin{tabular}{lcrrcc}
\hline \hline$\alpha$ & $\varepsilon$ & $L$ & $N$ & $K$ & $\delta K$ \\
\hline $2 / 3$ & $1 / 3$ & 3 & 3 & 0.7376 & $6 \times 10^{-4}$ \\
& & 6 & 12 & 0.5187 & $4 \times 10^{-4}$ \\
$4 / 9$ & $2 / 9$ & 3 & 2 & 0.4419 & $4 \times 10^{-4}$ \\
& & 6 & 8 & 0.4455 & $2 \times 10^{-4}$ \\
$8 / 25$ & $4 / 25$ & 5 & 4 & 0.3874 & $1 \times 10^{-4}$ \\
& & 10 & 16 & 0.3873 & $5 \times 10^{-5}$ \\
$1 / 4$ & $1 / 8$ & 4 & 2 & 0.3483 & $2 \times 10^{-4}$ \\
& & 8 & 8 & 0.3375 & $4 \times 10^{-5}$ \\
\hline \hline
\end{tabular}

\section{CREATION AND OBSERVATION}

Several laboratories currently have the technology to create a rotating optical lattice [20,21], which can be directly used to create the system described here. Those experiments still are far from the Mott regime, but they are progressing rapidly. An alternative approach to implementing Eq. (1) is to use a nonrotating lattice, and generate the phases on the hopping matrix elements by some other means [22]. The most advanced demonstration of this technique was from Lin et al. [23].

One of the more promising schemes for experimental observation of the incompressible states described here is through in situ imaging of the density profile of a trapped gas [4,24]. The fractional quantum Hall states should appear as extra steps in the density profile near the Mott insulator plateaus. Moreover, as the magnitude of the effective magnetic field increases, these steps move in predictable ways: the density is set by the magnetic flux, and the size of the gap (hence the spatial size of the plateau) increases with magnetic field. One can even imagine that for a fixed flux there will appear a sequence of FQH states with larger even denominators and thus with smaller densities all the way up to the MI-SF phase boundary; however, their size will be much smaller and they may not be discernible at all. Other probes for the FQH states may be noise correlations in time-of-flight experiments, measurement of the Hall conductance for the mass current in a tilted lattice, or Bragg spectroscopy [2-7,24].

A major impediment to observation of these states is the need to reduce the temperature to below the scale of the gap, which is a fraction of the hopping matrix element $t$. Such temperatures are currently hard to reach reliably.

\section{SUMMARY}

In summary, we have predicted that experiments on bosons in rotating lattices (or in lattices with an artificial gauge field) will see a phase where the excitations on top of a Mott insulator form a bosonic fractional quantum Hall state. We base our prediction on a set of variational calculations, supplemented by exact diagonalization of small systems. We find that the MI $+v=1 / 2$ Laughlin state has a lower energy than the Gutzwiller mean-field vortex lattice when the density of excess particles or holes, $\varepsilon=N / L^{2}$, is chosen appropriately $(\varepsilon=$ $\alpha \nu=\alpha / 2$ ), and the hopping $t$ is sufficiently small compared to the interactions $U$. In this regime, we find that the overlap between the exact ground state and the Laughlin state is as large as $96 \%$, but the overlap with the superfluid is smaller than $10 \%$. We produced a phase diagram (Fig. 4), illustrating where this phase should be found at low temperatures.

\section{ACKNOWLEDGMENTS}

R.O.U. is supported by TÜBİTAK. R.O.U. also wishes to thank Eliot Kapit for useful discussions and acknowledges the hospitality of the LASSP, Cornell University, where this work was completed. This material is based in part upon work supported by the National Science Foundation under Grant No. PHY-0758104.
[1] R. O. Umucalılar and M. Ö. Oktel, Phys. Rev. A 76, 055601 (2007).

[2] M. Hafezi, A. S. Sørensen, E. Demler, and M. D. Lukin, Phys. Rev. A 76, 023613 (2007).

[3] A. S. Sørensen, E. Demler, and M. D. Lukin, Phys. Rev. Lett. 94, 086803 (2005).

[4] R. N. Palmer, A. Klein, and D. Jaksch, Phys. Rev. A 78, 013609 (2008); R. N. Palmer and D. Jaksch, Phys. Rev. Lett. 96, 180407 (2006).

[5] R. Bhat, M. Krämer, J. Cooper, and M. J. Holland, Phys. Rev. A 76, 043601 (2007); R. Bhat, B. M. Peden, B. T. Seaman, M. Krämer, L. D. Carr, and M. J. Holland, ibid. 74, 063606 (2006); R. Bhat, L. D. Carr, and M. J. Holland, Phys. Rev. Lett. 96, 060405 (2006).

[6] G. Möller and N. R. Cooper, Phys. Rev. Lett. 103, 105303 (2009).

[7] N. R. Cooper, Adv. Phys. 57, 539 (2008).

[8] J. Ye and S. Sachdev, Phys. Rev. Lett. 80, 5409 (1998); D. Pfannkuche and A. H. MacDonald, Phys. Rev. B 56, R7100
(1997); F. F. Assaad and S. Biskamp, ibid. 51, 1605 (1995); W. Chen, M. P. A. Fisher, and Y.-S. Wu, ibid. 48, 13749 (1993); X.-G. Wen and Y.-S. Wu, Phys. Rev. Lett. 70, 1501 (1993); H. Aoki, ibid. 55, 1136 (1985); A. H. MacDonald, Phys. Rev. B 29, 6563 (1984); D. J. Thouless, M. Kohmoto, M. P. Nightingale, and M. den Nijs, Phys. Rev. Lett. 49, 405 (1982).

[9] D. R. Hofstadter, Phys. Rev. B 14, 2239 (1976).

[10] D. S. Goldbaum and E. J. Mueller, Phys. Rev. A 79, 021602(R) (2009); 77, 033629 (2008).

[11] M. Ö. Oktel, M. Niţă, and B. Tanatar, Phys. Rev. B 75, 045133 (2007).

[12] M. Niemeyer, J. K. Freericks, and H. Monien, Phys. Rev. B 60, 2357 (1999).

[13] R. B. Laughlin, Phys. Rev. Lett. 50, 1395 (1983).

[14] F. D. M. Haldane and E. H. Rezayi, Phys. Rev. B 31, 2529 (1985).

[15] I. Bloch, J. Dalibard, and W. Zwerger, Rev. Mod. Phys. 80, 885 (2008). 
[16] E. J. Mueller, T.-L. Ho, M. Ueda, and G. Baym, Phys. Rev. A 74, 033612 (2006).

[17] F. D. M. Haldane, Phys. Rev. Lett. 55, 2095 (1985).

[18] See, for instance, Quantum Monte Carlo Methods in Physics and Chemistry, edited by M. P. Nightingale and C. J. Umrigar, NATO Science Series, Series C: Mathematical and Physical Sciences, Vol. 525 (Kluwer Academic, Boston, 1999); S. Fahy, X. W. Wang, and S. G. Louie, Phys. Rev. B 42, 3503 (1990); N. Trivedi and D. M. Ceperley, ibid. 41, 4552 (1990); H. Yokoyama and H. Shiba, J. Phys. Soc. Jpn. 56, 1490 (1987).

[19] V. Ambegaokar and M. Troyer, Am. J. Phys. 78, 150 (2010).

[20] R. A. Williams, S. Al-Assam, and C. J. Foot, Phys. Rev. Lett. 104, 050404 (2010).

[21] S. Tung, V. Schweikhard, and E. A. Cornell, Phys. Rev. Lett. 97, 240402 (2006).
[22] K. J. Günter, M. Cheneau, T. Yefsah, S. P. Rath, and J. Dalibard, Phys. Rev. A 79, 011604(R) (2009); Q. Zhang, J. Gong, and C. H. Oh, ibid. 79, 043632 (2009); I. B. Spielman, ibid. 79, 063613 (2009); G. Juzeliūnas, P. Öhberg, J. Ruseckas, and A. Klein, ibid. 71, 053614 (2005); J. Ruseckas, G. Juzeliūnas, P. Öhberg, and M. Fleischhauer, Phys. Rev. Lett. 95, 010404 (2005); E. J. Mueller, Phys. Rev. A 70, 041603 (2004); D. Jaksch and P. Zoller, New J. Phys. 5, 56 (2003); J. Ruostekoski, G. V. Dunne, and J. Javanainen, Phys. Rev. Lett. 88, 180401 (2002).

[23] Y.-J. Lin, R. L. Compton, K. Jimenez-Garcia, J. V. Porto, and I. B. Spielman, Nature (London) 462, 628 (2009).

[24] R. O. Umucalılar, H. Zhai, and M. Ö. Oktel, Phys. Rev. Lett. 100, 070402 (2008). 